\title{
Preface
}

\section{His Bundle Pacing: Everything Old Is New Again}

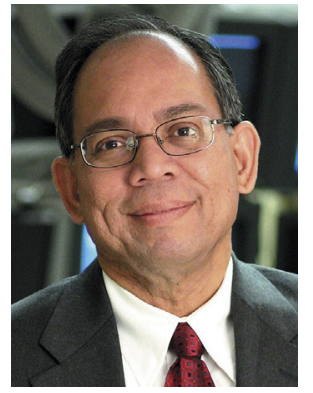

Pramod Deshmukh, MD

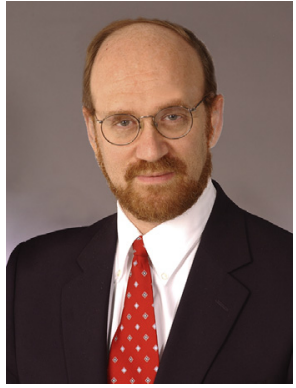

Kenneth A. Ellenbogen, MD

Editors

The development of His bundle pacing is both a story of technological advancement and a return to old physiologic principles. Electrical pacing of the heart began as an open-chest procedure performed by surgeons. It was only with the advent of the transvenous approach that cardiologists began to implant pacemakers. Subsequent developments then focused on improvements in technology with advancements in batteries, digital electronics, programming functions, and leads. During this time, the seminal work of Dr Wiggers from 1925, which identified the deleterious impact of ventricular pacing, appeared to be forgotten and pacing in the ventricular musculature remained the standard.

Reflecting on our early experience, I feel very lucky to be trained as an electrophysiologist prior to implanting pacemakers. Thanks to this background, an excellent physiology teacher, and perhaps the naiveté of an early operator, we first performed selective His bundle pacing with a desire to maintain normal ventricular activation. After establishing it was indeed possible, the challenges early on made it important to define selective His pacing. In addition to published criteria by Dr Sherlag, the third criteria (all-or-none) was defined and added. This third criterion is now labeled "single capture threshold" and forms the basis of select versus nonselect pacing.
Since this initial experience, His bundle pacing has proliferated and has been employed in diverse settings. The contributors to this issue deserve special praise for reproducing our observations with systematic study and then carrying them much further into new contexts. It is especially notable that the field of His bundle pacing has been driven primarily by clinicians seeking to best treat their patients rather than by funding from the medical industry. It is through their vigorous enthusiasm that $\mathrm{His}$ bundle pacing has become widespread, and there is now the development of specialized tools for its performance.

This text seeks to summarize the physiology of His bundle pacing, practical knowledge useful in its adoption, and the growing wealth of experience of utilizing His bundle pacing in various contexts. We hope that in its readings others will continue to adopt His bundle pacing into their practice and be inspired to join in advancing the field.

There are many people without whom this issue would not be complete. I would like to thank my medical school professor, Dr Dabholkar, for giving me a solid foundation in physiology. Personal encouragement by Dr Sherlag and the kind review by Dr Scheinman were also of immense help in the early days. I would like to thank Ken for his 
insights, contributions, and guidance for this issue. Last but not least, the contributors to this issue deserve a special thank you, as without their efforts it would not be published.

Pramod Deshmukh, MD Director, Arrhythmia Service Guthrie Health Care System

1 Guthrie Square Sayre, PA 18840, USA
Kenneth A. Ellenbogen, MD Division of Cardiovascular Diseases Virginia Commonwealth University Health Science Center 1200 East Marshall Street Gateway Building, 3rd Floor, 3-216 Richmond, VA 23298, USA

$$
\text { E-mail addresses: }
$$

prmode@yahoo.com (P. Deshmukh) ken.ellenbogen@gmail.com (K.A. Ellenbogen) 